\title{
The Role of Plasma Lactate Dehydrogenase Testing in the Prediction of Severe Conditions in Newborn Infants: A Prospective Study
}

This article was published in the following Dove Press journal: Research and Reports in Neonatology

\author{
Ton Nu Van Anh $\mathbb{D}^{1, *}$ \\ Tran Kiem Hao (iD ${ }^{2, *}$ \\ Huynh Huu Hoang ${ }^{3}$ \\ 'Department of Pediatrics, Hue \\ University of Medicine and Pharmacy, \\ Hue University, Hue City, Vietnam; \\ ${ }^{2}$ Pediatric Center, Hue Central Hospital, \\ Hue City, Vietnam; ${ }^{3}$ Quang Nam Hospital \\ for Women and Children, Tam Ky City, \\ Vietnam
}

*These authors contributed equally to this work
Correspondence: Ton Nu Van Anh

Department of Pediatrics, Hue University of Medicine and Pharmacy, Hue University, 06 Ngo Quyen St, Hue City, Vietnam Tel +84982066063

Email tonnuvananh@huemed-univ.edu.vn
Purpose: Plasma lactate dehydrogenase (LDH) is an indicator of body tissue hypoxia. This study aimed to determine the relationship between plasma lactate dehydrogenase concentrations and severe conditions in newborn infants.

Patients and Methods: A cross-sectional study was performed on newborn infants who were admitted to the newborn care unit at Hue University hospital from April 2016 to May 2017 in the early neonatal period (within 12 hours postpartum). Plasma LDH was measured at the time of admission and correlated to clinical conditions.

Results: In 275 newborn infants, plasma LDH levels in the term infants were significantly higher than that in the preterm infants [751 (IQR: 602-922) vs 594 (IQR: 496.75-767.25), $\mathrm{p}=0.0006]$. There was a relationship between the signs of feeding problems, tachypnea, and cyanosis with plasma LDH levels $(\mathrm{p}<0.01)$. Infants with asphyxia had significantly higher LDH values than the non-asphyxia group [756 (640-1110) vs $712(576-882.25) \mathrm{p}=0.0289]$. Infants with early-onset neonatal sepsis had significantly higher LDH values than those without early-onset neonatal sepsis [755.5 (IQR: 645-960.5) vs 707 (IQR: 562.25-881.25) $\mathrm{p}=0.0035]$. Infants with respiratory distress requiring continuous positive airway pressure (CPAP) had significantly higher LDH values than those with illnesses not requiring CPAP [903 (IQR: 628.75-1285.25) vs 719 (IQR: 576.5-882) p=0.0421]. By using multivariate regression analysis, we found a significant multifactorial correlation between gestational age, early-onset neonatal sepsis, asphyxia, and respiratory distress requiring CPAP with plasma LDH levels ( $\mathrm{p}<0.05)$.

Conclusion: Plasma LDH level can be a good marker for the prognosis of severe conditions in newborn infants, including early-onset neonatal sepsis, asphyxia, and respiratory-distress. Keywords: newborn infants, plasma lactate dehydrogenase, neonatal sepsis, asphyxia, respiratory distress

\section{Introduction}

Globally, there has been a downward trend in childhood mortality in recent decades, but neonatal mortality remains high. According to a report by the World Health Organization and the United Nations Children's Fund in 2015, the infant mortality rate is $19 \%$ o worldwide, and, in Vietnam, the rate was $11 \%$ in 2015. ${ }^{1}$ Neonatal mortality is different between countries because of their various geographic, economic, or social conditions. In Vietnam, although the mortality rate for children under five has decreased in recent years, a large number of children still die each year, mainly in the neonatal stage. Disorders in newborns are more challenging to detect early because their clinical signs are often 
nonspecific and diverse. In severe disorders, the oxygen concentration is deficient, glucose is metabolized by the anaerobic pathway, and pyruvate is then deoxidized into lactate by lactate dehydrogenase. ${ }^{2}$ Moreover, lactate dehydrogenase $(\mathrm{LDH})$ is an intracellular enzyme that responds to energy shortages in all organs. Therefore, plasma LDH is also an indicator of body tissue hypoxia. Some previous studies on the LDH levels in severe neonates (reported by Karlsson et $\mathrm{al}^{3}{ }^{3}$ Reddy et $\mathrm{al},{ }^{4}$ and Sanjay et al $)^{5}$ have concluded that there is a correlation between LDH concentrations and asphyxia in neonates. A study by Ozkiraz et al on respiratory distress in neonate also showed a relationship between LDH levels and the duration of supplying oxygen. ${ }^{6}$ Although there are a few studies on $\mathrm{LDH}$, they have not provided an overall picture of the role of LDH in severe disorders in neonates.

This study aimed to determine the relationship between plasma lactate dehydrogenase concentration and severe conditions in newborn infants. The findings of the study in a clinical context, especially if LDH test help to guide further neonatal intensive care.

\section{Patients and Methods}

This prospective study was carried out in newborn infants who were admitted to the newborn care unit at the Hue university hospital from April 2016 to May 2017 under the approval of the hospital Ethical Commette with the reference number 01042016/HUH. Informed consent was obtained from the patient's parents. A full history was collected as protocol and entered into the clinical research database.

All study subjects were examined and evaluated for their gestational age (new Ballard score) and nutritional level to classify the newborns (Fenton). The maternal history, clinical symptoms, and signs of serious illness were also recorded, including poor or no breastfeeding, fever, hypothermia, umbilical infection, and shortness of breath. Some severe conditions, including hypoxia (APGAR $\leq 7)$, neonatal sepsis (ANAES criteria), and neonatal respiratory distress syndrome (requiring continuous positive airway pressure), were diagnosed accordingly.

Plasma LDH testing was done at the time of admission, within 12 hours postpartum. Two milliliters of serum with Li-heparin and plasma underwent hemolysis by the automated analyzed technique in a COBAS 600 (501) machine with Reagent A (400 mM methylglucamine (MEG) and 61 $\mathrm{mM}$ lactate solution; $\mathrm{pH}$ 9.437C) and Reagent B (a solution containing $61 \mathrm{mM} \mathrm{NAD)}$.
Data processing employing statistical methods was performed using the Medcalc 13.0 software. Qualitative variables, such as gender, gestational age, risk factors for early neonatal infection, signs of severe illness, and severe neonatal pathologies, are presented by frequency and percentage. Quantitative variables like LDH and weight are presented by the median, 25 th percentile, 75 th percentile, or mean index, and standard deviation.

We used Spearman's rank correlation coefficient to consider the linear correlation between the two quantitative variables, $\mathrm{LDH}$, and leukocytes. A multivariate regression analysis was used to find the factors associated with plasma LDH concentration through the following steps: selecting variables, exploring each variable, removing unimportant variables, checking interactions between variable numbers, and finding the optimal model.

\section{Results}

From April 2016 to May 2017, a total of 275 neonates in the Newborn Care Unit of Hue University Hospital were recruited $(57.1 \%$ male and $42.9 \%$ female). Full-term infants accounted for $79.3 \%$, and preterm infants accounted for $20.7 \%$. The general characteristics of the study group of neonates are shown in Table 1.

Table I General Characteristics of the Study Group of Newborn Infants

\begin{tabular}{|l|l|l|}
\hline Variables & $\mathbf{n}$ & $\%$ \\
\hline $\begin{array}{l}\text { Gestational age } \\
\text { Preterm infants }\end{array}$ & $(\mathrm{n}=57)$ & 20.7 \\
Full-term infants & $(\mathrm{n}=218)$ & 79.3 \\
\hline $\begin{array}{l}\text { No breast-feeding or poor feeding } \\
\text { Yes }\end{array}$ & $(\mathrm{n}=47)$ & \\
No & $(\mathrm{n}=228)$ & 17 \\
\hline $\begin{array}{l}\text { Shortness of breath } \\
\text { Yes }\end{array}$ & $(\mathrm{n}=103)$ & 37.4 \\
No & $(\mathrm{n}=172)$ & 62.6 \\
\hline Asphyxia & & \\
Yes & $(\mathrm{n}=22)$ & 8 \\
No & $(\mathrm{n}=253)$ & 92 \\
\hline Early neonatal sepsis & & \\
Yes & $(\mathrm{n}=72)$ & 26.2 \\
No & $(\mathrm{n}=203)$ & 73.8 \\
\hline Respiratory distress requiring CPAP & & \\
Yes & $(\mathrm{n}=1 \mathrm{I})$ & 4 \\
No & $(\mathrm{n}=264)$ & 96 \\
\hline
\end{tabular}


Table 2 The Relationship Between LDH Concentration and Newborn Infant Disorders

\begin{tabular}{|l|l|l|}
\hline Variables & $\begin{array}{l}\text { LDH (U/I) } \\
\text { Median, (IQR) }\end{array}$ & Plog \\
\hline $\begin{array}{c}\text { Gestational age } \\
\text { Preterm infants }(n=57) \\
\text { Full-term infants }(n=218)\end{array}$ & $\begin{array}{l}594(496.75-767.25) \\
751(602-922)\end{array}$ & $P=0.0006$ \\
\hline $\begin{array}{l}\text { No breast-feeding or poor feeding } \\
\text { Yes }(n=47)\end{array}$ & $823(656.25-952)$ & $P=0.044 \mid$ \\
No $(n=228)$ & $706(568-872.5)$ & \\
\hline $\begin{array}{l}\text { Shortness of breath } \\
\text { Yes }(n=103)\end{array}$ & $752(616.5-946.7)$ & $P=0.0198$ \\
No $(n=172)$ & $701.5(567-870.5)$ & \\
\hline $\begin{array}{l}\text { Asphyxia } \\
\text { Yes }(n=22)\end{array}$ & $756(640-1110)$ & $P=0.0289$ \\
No $(n=253)$ & $712(576-882.25)$ & \\
\hline $\begin{array}{l}\text { Early neonatal sepsis } \\
\text { Yes }(n=72)\end{array}$ & $755.5(645-960.5)$ & $P=0.0035$ \\
No $(n=203)$ & $707(562.25-881.25)$ & \\
\hline $\begin{array}{l}\text { Respiratory distress requiring CPAP } \\
\text { Yes }(n=11)\end{array}$ & $903(628.75-1285.25)$ & $P=0.0421$ \\
No $(n=264)$ & $719(576.5-882)$ & \\
\hline
\end{tabular}

Note: Plog: Independent $t$-test.

The relationship between LDH concentration and newborn disorders is shown in Table 2. Plasma LDH levels were higher in the full-term infants than in the preterm infants [751 U/1 (IQR: 602-922) vs $594 \mathrm{U} / 1$ (IQR: 496.75-767.25), $\mathrm{p}=0.0006]$. When comparing the infants with severe clinical signs to those with no signs of severe disease, the infants with signs of no breastfeeding or poor feeding had higher LDH levels in the plasma than those without these signs [823 U/1 (IQR: 656.25-952) vs 706 U/1 (IQR: 568-872.5)]. Infants with signs of shortness of breath had higher plasma LDH levels than those with no signs [752 U/1 (IQR: 616.5946.7) vs 701.5 U/1 (IQR: 567-870.5)]. In our study, the plasma LDH levels in infants with early neonatal infection were higher than those in infants who did not have early neonatal sepsis [755.5 U/l (IQR: 645-960.5) vs 707 U/1 (IQR: 562.25-881.25) $\mathrm{p}=0.0035$ ]. The plasma LDH levels of infants with asphyxia [756 U/1 (IQR: 640-1110)] were higher than those who are not asphyxiated [712 U/1 (IQR: 576-882.25)]. The levels of infants with respiratory failure needing continuous positive airway pressure were higher than those without respiratory failure and no need to breathe CPAP [903
Table 3 The Model Optimally Predicting the Factors Related to Plasma LDH Levels Through a Multivariate Regression Analysis

\begin{tabular}{|l|l|l|l|}
\hline Factors & $\mathbf{p}$ & $\mathbf{r}$ & $\mathbf{R}^{2}$ \\
\hline Gestational age & 0.0004 & 0.1822 & 0.09116 \\
Asphyxia & 0.0285 & 0.1420 & \\
Early neonatal infection & 0.0170 & 0.1811 & \\
Respiratory distress requiring CPAP & 0.0047 & 0.1291 & \\
\hline
\end{tabular}

U/1 (IQR: 628.75-1285.25) vs 719 U/1 (IQR: 576.5-882) $\mathrm{p}=0.0421]$.

There was a correlation between gestational age, early neonatal infection, asphyxia, and respiratory distress requiring CPAP with plasma LDH levels with a statistically significant association ( $\mathrm{p}<0.05$; $\mathrm{R} 2=$ 0.09116 , meaning 9.116\%) (Table 3). Changes in plasma LDH levels were attributed to changes in the gestational age, early neonatal sepsis, asphyxia, and respiratory distress requiring CPAP.

\section{Discussion}

In severe illness, the body lacks the oxygen and glucose that is metabolized in the anaerobic pathway, and pyruvate is oxidized to lactate by the lactate dehydrogenase. The higher the oxygen deficiency, the greater the anaerobic metabolism, so, as more lactate is produced, the LDH also increases. ${ }^{2}$ The study of Ozkiraz et al showed a positive correlation between the duration of oxygen with lactate and lactate dehydrogenase (LDH) levels. LDH offered the best predictive value for prolonged oxygen requirements with a positive predictive value (PPV) of $88.9 \%$. The predictive value of lactate exceeded the predictive value of $\mathrm{LDH}$, aspartate aminotransferase, and the percentage of normoblasts needed to predict the requirements of respiratory support with a PPV of $88.5 \% .{ }^{6}$ In the study of Karlsson et al, enzymes were measured within 12 hours postpartum in newborn infants with a differing degree of hypoxic-ischemic encephalopathy (HIE) $(n=$ 41) and infants with signs of fetal distress during birth $(n=205)$ without HIE (non-HIE group). All infants were randomized into two groups. One group $(n=123)$ was used to calculate the cut off limits for the enzymes studied, and the other group $(n=123)$ was used to calculate the predictive value of the enzymes for the detection of HIE. Using ROC curves, a cut off level of $1049 \mathrm{U} / \mathrm{L}$ for [LDH] was chosen as the best predictor of HIE (sensitivity $100 \%$ and specificity $97 \%$ ) but also for long term outcomes after HIE. $^{3}$ 
In the study group of 275 newborn infants at Hue University Hospital, the median of the plasma LDH levels was $719 \mathrm{U} / 1$, the 25 th percentile was $578.25 \mathrm{U} / 1$, and the 75th percentile was $892.5 \mathrm{U} / \mathrm{l}$. In a study by Matsuoka et al, the value of the plasma LDH in infants from 1 to 3 days old was found to be $623.6 \mathrm{U} / \mathrm{l}^{7}$ In the Eva study, the 25th and 75th percentiles of intravenous cord plasma LDH were $252-636 \mathrm{U} / \mathrm{L} .{ }^{8}$ Thus, our study showed an average LDH level higher than the normal value of plasma LDH.

In our study, the full-term infants had significantly higher levels of LDH than the preterm infants with a statistical significance of $p=0,0006$. Zanardo's study of intracellular enzymes in preterm and full-term infants showed lower levels of glutamic-oxaloacetic transaminase and glutamic-pyruvic transaminase activity in preterm infants. LDH is also an intracellular enzyme. In our study, the plasma LDH levels were also lower in preterm infants. This is explained by the fact that the cell membranes with cell hypoxia in preterm infants are more persistent and that cell metabolism is more incomplete in preterm infants compared to full-term and post-term infants. $^{9}$

When comparing infants with severe clinical signs to those without signs, infants with signs of no breast-feeding or poor feeding had higher plasma LDH levels than those without signs of breast-feeding or poor feeding [823 (656.25-952); 706 (568-872.5), $\mathrm{P}=0.0441$ ]. Infants with signs of shortness of breath had higher plasma LDH levels than those with no signs of rapid onset [752 (616.5-946.7); 701.5 (567-870.5); $\mathrm{P}=0.0198$ ].

According to a study by Karlsson et al, infants with respiratory symptoms, such as coughing, wheezing, or rapid breathing, showed no difference $(p=0.05)$ between the group requiring active neonatal care and that requiring no active neonatal care or between the group with no breastfeeding and that featuring poor feeding and fever. The plasma LDH levels in the seronegative neonatal intensive care groups were also higher than those without active neonatal care. ${ }^{10}$

For asphyxia, the LDH of the asphyxiated group (with $756(640-1110) \mathrm{U} / \mathrm{l})$ was higher than that of the nonasphyxiated infants (with 712 (576-882.25) U/l), which was statistically significant at $p<0.05$. Reddy's study concluded that the plasma LDH levels of infants at 72 hours of age most accurately distinguish asphyxiated newborn infants from asymptomatic asphyxiated neonates. ${ }^{4}$ Sanjay's study had a mean cut-off LDH value of $580 \mathrm{U} / 1$ with a sensitivity of $59.18 \%$ and a specificity of $92 \% .^{5}$
Another study on asphyxia and HIE by Choudhary et al also showed LDH results at 72 hours old as a tool for neonatal asphyxia diagnosis and LDH elevation related to HIE levels. There is, moreover, a difference in the LDH levels between HIE degree 0 and degree II, HIE degrees 0 and III, HIE degrees I and II, and HIE degrees I and III. ${ }^{11}$ A study by Karlsson et al showed that THE LDH within the first 12 hours postpartum is a good predictor of HIE levels. The LDH cut-off of $1049 \mathrm{U} / 1$ is the best predictor, with an HIE sensitivity of $100 \%$ and a specificity of $97 \%{ }^{3}$

For early neonatal sepsis, in our research, the plasma LDH of the infants with early neonatal infection (755.5 (645-960.5) U/1) was higher than that for those without early neonatal infections (707 (562.25-881.25) U/1), which was statistically significant at $p<0.01$. There are several studies on blood LDL levels in various infections. Morini's study concluded that blood LDL levels were significantly increased in infants with necrotizing enterocolitis $(p<0.005) .{ }^{12}$ A study by Powers et al found that peripheral plasma LDH levels increased in infants with bacterial meningitis. The leukocyte LDH activity in patients with bacterial meningitis was significantly higher than that in patients with nonbacterial meningitis $(p<0.01)$ and the normal controls $(\mathrm{p}<0.001) .{ }^{13}$ A study by Zein el al. concluded that elevated plasma LDH levels in severe infections, as a marker of tissue damage, did not improve LDH levels at 48 hours as a strong predictor of mortality in patients with severe sepsis. Death was associated with a higher LDH level $(656 \pm 79 ; \mathrm{p}<0.001$ vs $369 \pm 72 \mathrm{U} / \mathrm{L})$. LDH levels that increase 48 hours after ICU admission (observed in 37 of 82 patients) were sensitive (0.72) and specific $(0.77)$ in predicting mortality. ${ }^{14}$

In our research, the plasma LDH levels of infants with respiratory distress requiring CPAP (903 (628.75-1285.25) $\mathrm{U} / \mathrm{l})$ was higher than the levels in those without respiratory distress requiring CPAP (719 (576.5-882) U/l), with statistical significance at $p<0.05$. A study by Ozkiraz et al correlated the duration of oxygen support with LDH concentrations ( $\mathrm{p}<0.01)$. With an LDH $750 \mathrm{U} / 1$ cut-off point, the best estimate for oxygen demand is $>72 \mathrm{~h}$, with a specificity of $90.6 \%$, a sensitivity of $47.6 \%$, and an area under the curve of $73.3 \%{ }^{6}$ CPAP is a non-invasive respiratory support method that is used for patients with respiratory failure and provides oxygen by maintaining continuous positive airway pressure throughout the respiratory cycle. A study by Ozkiraz et al concluded that there is a link between plasma LDH levels and the need to receive oxygen in the hospital. Thus, the higher 
plasma LDH levels in infants who need CPAP (higher than those without CPAP) is not difficult to explain. ${ }^{6}$

Building a model of the optimal predictors of plasma LDH-related factors through a multivariable regression analysis, we found that there is a correlation between the four factors and plasma LDH levels in the multidisciplinary relationship between gestational age, early neonatal infection, asphyxia, and CPAP respiratory failure at $\mathrm{p}<0.05\left(\mathrm{R}^{2}=0.09116\right)$, meaning that $9.116 \%$ showed changes in their plasma LDH levels that were attributable to changes in gestational age, early neonatal sepsis, asphyxia, and respiratory distress requiring CPAP.

In conclusion, there is a correlation, although very weak, between the plasma LDH levels with early neonatal infection, asphyxia, and respiratory distress requiring CPAP in neonates admitted to the intensive care unit. Because of the wide variability of test results for $\mathrm{LDH}$, an additional study would be necessary to use LDH as a predictive biomarker. Of all the diseases considered, the association of LDH levels and hypoxic-ischemic encephalopathy severity seems the most promising, especially for the low-resource medical settings. This would be reasonable for additional studies in this area.

\section{Ethics}

The study was approved by the ethics committee of Hue University hospital (Hue city, Vietnam). Informed consent was obtained from all patient's parents. The study was in accordance with the Declaration of Helsinki.

\section{Disclosure}

The abstract of this paper was presented at the 23rd Annual Congress on Pediatrics \& Neonatology (November 05-06, 2018 Bangkok, Thailand) as a poster presentation with interim findings. The poster's abstract was published in 'Poster Abstracts' in Pediatrics \& Therapeutics 2018, Volume 8 DOI: 10.4172/2161-0665-C9-076.

The authors report no funding and no conflicts of interest for this work.

\section{References}

1. WHO, Unicef, World Bank, UN-DESA Population Division. Levels \& Trends in Child Mortality. 8; 2015.

2. Burtis CA, Ashwood ER, Bruns DE. Lactate dehydrogenase. Tietz Textbook Clin Chem Molecular Diagnostics. 2012;590-591.

3. Karlsson M, Wiberg-Itzel E, Chakkarapani E, Blennow M, Winbladh B, Thoresen M. Lactate dehydrogenase predicts hypoxic ischaemic encephalopathy in newborn infants: a preliminary study. Acta Paediatr. 2010;99(8):1139-1144. doi:10.1111/j.1651-2227.20 10.01802.x

4. Reddy S, Dutta S, Narang A. Evaluation of lactate dehydrogenase, creatine kinase and hepatic enzymes for the retrospective diagnosis of perinatal asphyxia among sick neonates. Indian Pediatr. 2008;45 (2):144-147.

5. Sanjay KM, Sarasu M, Sulekha C, Vijayalakshmi M. Evaluation of serum creatine kinase muscle-brain fraction (CK-MB) and lactate dehydrogenase (LDH) as markers of perinatal asphyxia in term neonates. Int J Med Health Sci. 2014;3(3):190-194.

6. Ozkiraz S, Gokmen Z, Boke SB, Kilicdag H, Ozel D, Sert A. Lactate and lactate dehydrogenase in predicting the severity of transient tachypnea of the newborn. $j$ Maternal-Fetal Neonatal Med. 2013;26 (12):1245-1248. doi:10.3109/14767058.2013.776532

7. Matsuoka S. Studies on lactic dehydrogenase (LDH) during development. 1. Changes of LDH activity and isozyme patterns in healthy human serum during development. Acta Paediatrica Japonica. 1968;10(1):13-21. doi:10.1111/j.1442-200X.1968.tb02643.x

8. Eva WI, Hampus J, Nana W, Linus O, Birger W, Mathias K. Lactic dehydrogenase in umbilical cord blood in healthy infants after different modes of delivery. J Neonatal Biol. 2015;4:1-4.

9. Zanardo V, Bondio M, Perini G, Temporin GF. Serum glutamic-oxaloacetic transaminase and glutamic-pyruvic transaminase activity in premature and full-term asphyxiated newborns. Biol Neonate. 1985;47(2):61-69. doi:10.1159/000242092

10. Karlsson M, Dung KT, Thi TL, et al. Lactate dehydrogenase as an indicator of severe illness in neonatal intensive care patients: a longitudinal cohort study. Acta Paediatr. 2012;101(12):12 25-1231. doi:10.1111/apa.12014

11. Choudhary M, Sharma D, Dabi D, Lamba M, Pandita A, Shastri S. Hepatic dysfunction in asphyxiated neonates: prospective case-controlled study. Clin Med Insights Pediatr. 2015;9:1-6. doi:10.4137/CMPed.S21426

12. Morini F, Ronchetti MP, Bagolan P. Increased serum activity of lactate dehydrogenase in infants with necrotising enterocolitis: a potential marker of advanced disease. Paediatr Child Health. 2009;19:1. doi:10.1016/j.paed.2009.05.024

13. Powers DW, Ayoub EM. Leukocyte lactate dehydrogenase in bacterial meningitis. Pediatrics. 1974;54(1):27-33.

14. Zein JG, Lee GL, Tawk M, Dabaja M, Kinasewitz GT. Prognostic significance of elevated serum lactate dehydrogenase (LDH) in patients with severe sepsis. Chest. 2004;126:873. doi:10.1378/ chest.126.4_MeetingAbstracts.873S

Research and Reports in Neonatology

Dovepress

Publish your work in this journal

Research and Reports in Neonatology is an international, peerreviewed, open access journal publishing original research, reports, editorials, reviews and commentaries on neonatal health. The manuscript

management system is completely online and includes a very quick and fair peer-review system. Visit http://www.dovepress. com/testimonials.php to read real quotes from published authors.

Submit your manuscript here: http://www.dovepress.com/research-and-reports-in-neonatology-journal 